\title{
Down-regulation of FTX promotes the differentiation of osteoclasts in osteoporosis through the Notch1 signaling pathway by targeting miR-137
}

Yingfeng Yu, Peiquan Yao, Zhikun Wang and Wenwei Xie (D)

\begin{abstract}
Background: Osteoporosis (OP) is one of the commonly seen bone diseases with low bone mineral densities and trauma fractures. Accumulative studies have demonstrated that the occurrence of OP is closely related to osteoclasts differentiation. LncRNA FTX has been demonstrated to inhibit the development of some human cancers. However, its potential functions in human OP remains to be elusive.

Methods: The expressions of FTX and miR-137 in bone and serum samples of patients with or without OP were measured. Bioinformatics analysis, RIP assays and luciferase reporter assays were performed to examine the upstream and downstream transactional factors of miR-137. Functional assays were conducted to check the roles of the Notching1 signaling pathway OP.

Results: FTX was suppressed in OP samples and serums, however, miR-137 was greatly elevated. FTX reduced osteoclast-genesis and inhibited osteogenic differentiation by targeting miR-137. This also inhibited the Notch1 signaling pathway.
\end{abstract}

Conclusion: Our experiments and results pointed out that IncRNA FTX up-regulated miR-137 in OP through the Notch1 signaling pathway.

Keywords: FTX, Osteoclasts, Notch1 signaling pathway, miR-137

\section{Background}

Osteoporosis (OP) is one of the commonly seen bone diseases with low bone mineral densities and trauma fractures [12, 13]. A large population suffers from OP, therefore, it possesses a great burden on both public health and the economy [18]. Accumulative studies have demonstrated that the occurrence of OP is closely related to osteoclasts differentiation [26]. In recent years, long non-coding RNAs (lncRNAs) and microRNAs

\footnotetext{
*Correspondence: in3740@163.com
Department of Orthopedics, the Third People's Hospital of Dongguan City,

* Correspondence: in3740@163.com No.1 Xianglong Road, Huangzhou, Shilong Town, Dongguan 523326, Guangdong Province, China
}

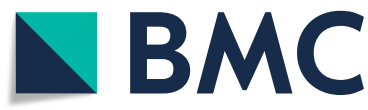

(c) The Author(s). 2020 Open Access This article is licensed under a Creative Commons Attribution 4.0 International License, which permits use, sharing, adaptation, distribution and reproduction in any medium or format, as long as you give appropriate credit to the original author(s) and the source, provide a link to the Creative Commons licence, and indicate if changes were made. The images or other third party material in this article are included in the article's Creative Commons licence, unless indicated otherwise in a credit line to the material. If material is not included in the article's Creative Commons licence and your intended use is not permitted by statutory regulation or exceeds the permitted use, you will need to obtain permission directly from the copyright holder. To view a copy of this licence, visit http://creativecommons.org/licenses/by/4.0/ The Creative Commons Public Domain Dedication waiver (http://creativecommons.org/publicdomain/zero/1.0/) applies to the data made available in this article, unless otherwise stated in a credit line to the data.

(miRNAs) are attracting more and more interest. These two non-coding RNAs may contribute to the dysregulated osteoclasts differentiation [8]. Therefore, biologists are paying more effort in the study of lncRNAs and miRNAs in the diagnostics, treatment directions, and prognostics for patients with OP.

LncRNAs are a group of RNA molecules with lengths of over 200 nucleotides, while they cannot be translated to proteins [11]. There is growing evidence suggesting that lncRNAs act as essential roles in a series of biological procedures including transcriptional regulations, cell apoptosis, as well as tumorigenesis [2, 6]. For OP, lncRNAs were proved to participate in the 
differentiation of osteoclasts. Long non-coding RNA FTX is a conserved functional lncRNA that could encode in the X-inactivation center [28]. LncRNA FTX has been demonstrated to inhibit the development of hepatocellular carcinoma [28], cardiomyocyte [17], and colorectal cancer [7]. However, its potential functions in human OP remains to be elusive.

miR-137 is a critical member of the miRNAs family, which has a length of around 20 nucleotides [20, 22]. Early in the year of 2008, J. Silber et al. indicated that miR-137 inhibited the proliferation of glioblastoma multiforme cells and induced differentiation of brain tumor stem cells [20]. There is also other literature revealing that miR-137 is playing an important role in a variety of human cancers $[16,29]$. In our preliminary experiments based on bioinformatics, we found that IncRNA FTX might bind with miR-137 in OP. Therefore, we are inspired to investigate whether FTX and miR-137 play a combined role in the development of OP. Previous studies have indicated that Notch signaling pathways have crucial roles in osteoclasts differentiation [14, 15]. Herein, we also examined if this signaling is involved in the development of OP. Thorough experiments and analysis were carried out to investigate the associations among lncRNA FTX, miR-137, and Notch1 signaling pathway.

\section{Methods}

\section{Primary human samples}

Patients with hip fractures at the third People's Hospital of Dongguan City were enrolled in this study, who were divided into 2 groups of OP patients and non-OP patients (control group). Osteoporosis were defined according to WHO criteria for classification of osteopenia and osteoporosis [12]. To be included, the fracture of the patients must meet the definition of fragility fracture caused by falling or low energy instead of violence or high-energy traumata. Patients with liver and kidney diseases; osteoarthritis; metabolic bone disease, ovarian diseases or other endocrine diseases were excluded. Also, patients underwent hormone therapy in recent 3 months or other chronic corticosteroid use were excluded due to the influence of these drugs on lncRNA and miRNA expression. Postmenopausal women with OP fractures $(n=40)$ and healthy volunteers $(n=40)$ were selected, who received hip replacement surgery in the hospital. Supplementary file 2 showed the patients' background regarding their age, gender, and BMI. RNA was extracted from the whole bone. According to the T-score of BMD, the patients were divided into two groups: the normal group (T-score $\geq-1.0$ ) and the OS group ( $\mathrm{T}$ score $\leq-2.5)$. Serum and bone samples were placed in endoprosthesis. All patients signed informed consent.
This research was approved by the Ethics Committee of the third People's Hospital of Dongguan City.

\section{CD14 + PBMC culture}

Peripheral blood mononuclear cells (PBMC) had extraction according to Sorensen et al. [21]. CD14 antibody magnetic cell sorting (Miltenyi, Germany) was utilized to purify CD14 + PBMCs. CD14 + PBMCs were seeded in at 250, 000 cells/well. Cells were growing in alpha minimum essential medium (Invitrogen, USA) with 10\% FBS (Gibco, CA), $50 \mathrm{IU} / \mathrm{mL}$ penicillin, and $50 \mathrm{mg} / \mathrm{mL}$ streptomycin, at $37^{\circ} \mathrm{C}$ with humidity and $5 \% \mathrm{CO}_{2}$. The culture medium was added in $25 \mathrm{ng} / \mathrm{mL}$ MCSF and 25 ng/mL RANKL (R\&D Systems, USA).

\section{Cell transfections}

FTX was amplified and purified. Then, pcDNA3.1 and FTX established the pcDNA-FTX recombinant plasmid and cloned to E. coli. The positive clones were selected and amplified. Next, recombinant plasmids were extracted and transfected to CD14+PBMCs. For overexpression miR-137, CD14+ PBMCs were transfected with miR-137 mimic, or controls with Lipofectamine 2000 (Invitrogen, USA). All plasmids were made from Genepharma, China. At $48 \mathrm{~h}$ post-transfection, the cells were used for following experiments.

\section{Cell proliferation assays}

4, 000 cells were seeded to a well from 96-well plate and cultured in alpha minimum essential medium with $10 \%$ FBS and antibiotics. $10 \mu \mathrm{L}$ CCK- 8 was added. We observed the signals at $2 \mathrm{~h}$ after the culture at $450 \mathrm{~nm}$.

\section{qRT-PCR}

Total RNA was extracted by TRIzol (Invitrogen, USA). $0.5 \mu \mathrm{g}$ RNA was reverse-transcribed by PrimeScript (Takara Bio, Japan). $1 \mu \mathrm{g}$ cDNA was employed to detect mRNA expressions by qRT-PCR with SYBR Kit (Takara Bio, Japan). GAPDH and U6 were regarded as references. Primers sequences are in the below:

GAPDH (forward: 5' -TGGATTTGGACGCATTGG TC-3' and reverse: $5^{\prime}$-TTTG.

CACTGGTACGTGTTGAT-3'); TNFRSF1B (forward: 5' -CGGGCCAACATGCAAAA. GTC-3' and reverse: $5^{\prime}$-CAGATGCGGTTCTGTTCCC-3'); ACP5 (forward: 5'-GACT.

GTGCAGATCCTGGGTG-3' and reverse: 5' -GGTCAGAG AATACGTCCTCAAA.

G-3');

MMP-9 (forward: 5'-TGTACCGCTATGGTTACACTCG-

$3^{\prime}$ and reverse: $5^{\prime}$-GG.

CAGGGACAGTTGCTTCT-3');

MMP-2 (forward: 5'-TGACTTTCTTGGATCGGGT. 


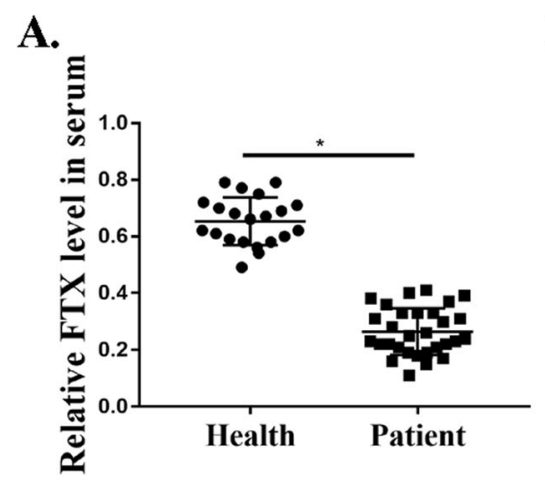

C.

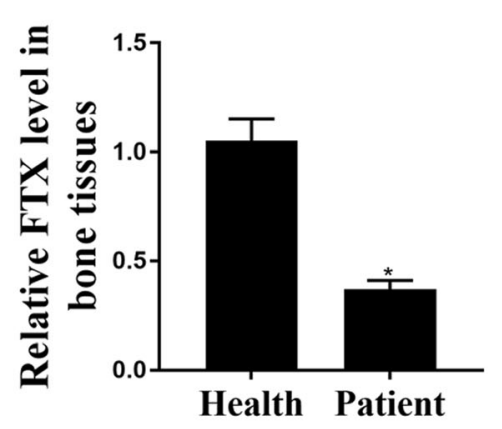

B.

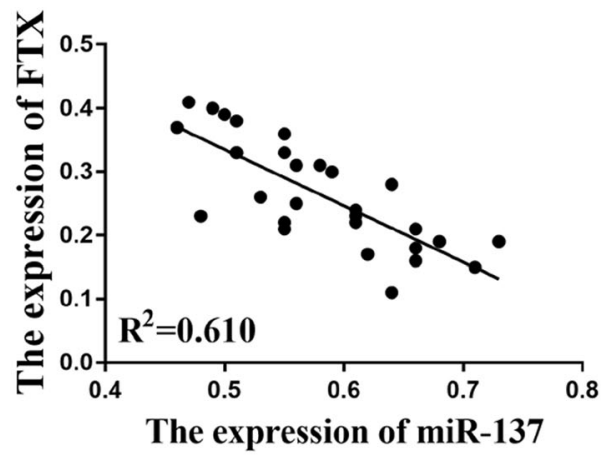

D.

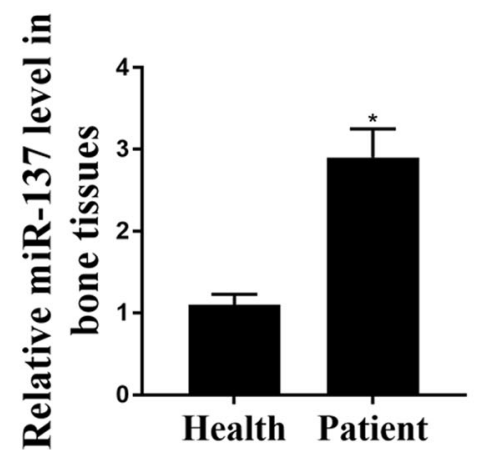

Fig. 1 FTX was down-regulated and miR-137 was up-regulated in osteoporotic samples. a. The FTX expressions in the serum of non-OP (healthy) $(n=21)$ and OP patients $(n=30)$. $\mathbf{b}$. The relationship between FTX and miR-137 expressions in the serum of OP patients. $\mathbf{c}$. The FTX expressions in the bone tissues of non-OP (healthy) $(n=7)$ and OP patients $(n=7)$. D. The miR-137 expressions in the bone tissues of non-OP (healthy) ( $n=7)$ and OP patients $(n=7) .{ }^{*} p<0.001$

CG-3' and reverse: 5' ${ }^{\prime}$-AAGCACCACATCAG ATGACTG-3');

TRAP (forward: 5' -TC.

ACCCTGACCTATGGTGC-3' and reverse: $5^{\prime}$ GCCGGACTCCAATGTTAAAGC-3');

cathepsin K (forward: 5'-CTGGCTGGGGTTAT GTCTCAA-3' and reverse: 5'-GGCT.

ACGTCCTTACACACGAG-3').

\section{Luciferase reporter assays}

FTX-WT (wild-type), FTX-MUT (mutant), Notch1-WT, Notch1-MUT were designed via the clone of WT 3' UTR or MUT 3'UTR to pMir-reporter vector (Ambion, USA). CD14 + PBMCs had co-transfections with luciferase plasmid, miR-137 mimic or miR-NC by Lipofectamine 2000. Dual-luciferase reporter assays (Promega, USA) were used to identify their binding effects.

\section{RNA pull-down assays}

For pull-down assay, miR-137 without complementary sites with Notch1 was seen as internal reference (termed NC). MiR-137, miR-137-Mut, and NC were labeled with biotin to generate Bio-miR-137, BiomiR-137-Mut, and Bio-NC by GenePharma Company (Shanghai, China). Thereafter, they were transfected into CD14 + PBMCs.
Forty-eight hours later, cells were harvested and incubated with Dynabeads M-280 Streptavidin (Invitrogen) for $10 \mathrm{~min}$. After washing three times with buffer solution, the enrichments of bound RNAs were quantified and analyzed by qRT-PCR.

\section{RNA immunoprecipitation (RIP)}

RIP assay was performed using the RNA-Binding Protein Immunoprecipitation Kit (Millipore). Firstly, we lysed CD14 + PBMCs and incubated them with AGO2 antibody or IgG. RNA-protein complexes were immunoprecipitated with protein A agarose beads. Next, we extracted the RNAs using Trizol (Invitrogen, USA). The IP-western was employed to measure AGO2 protein and qRT-PCR was conducted to detect the mRNA expression of FTX, using RIPAb+ Ago2-RIP Validated Antibody and Primer Set, 03-110, Merck Millipore, US.

\section{TRAP staining assay}

Induced osteoclasts were fixed with $4 \%$ paraformaldehyde (PFA) for $20 \mathrm{~min}$ at room temperature and then stained by TRAP fluid for $1 \mathrm{~h}$. TRAP solution was prepared by mixing acetate buer (pH 5.0), $50 \mathrm{mM}$ sodium tartrate, naphthol AS-MX phosphate (Sigma Aldrich, Tokyo, Japan), and Fast Red Violet LB salt (Sigma 

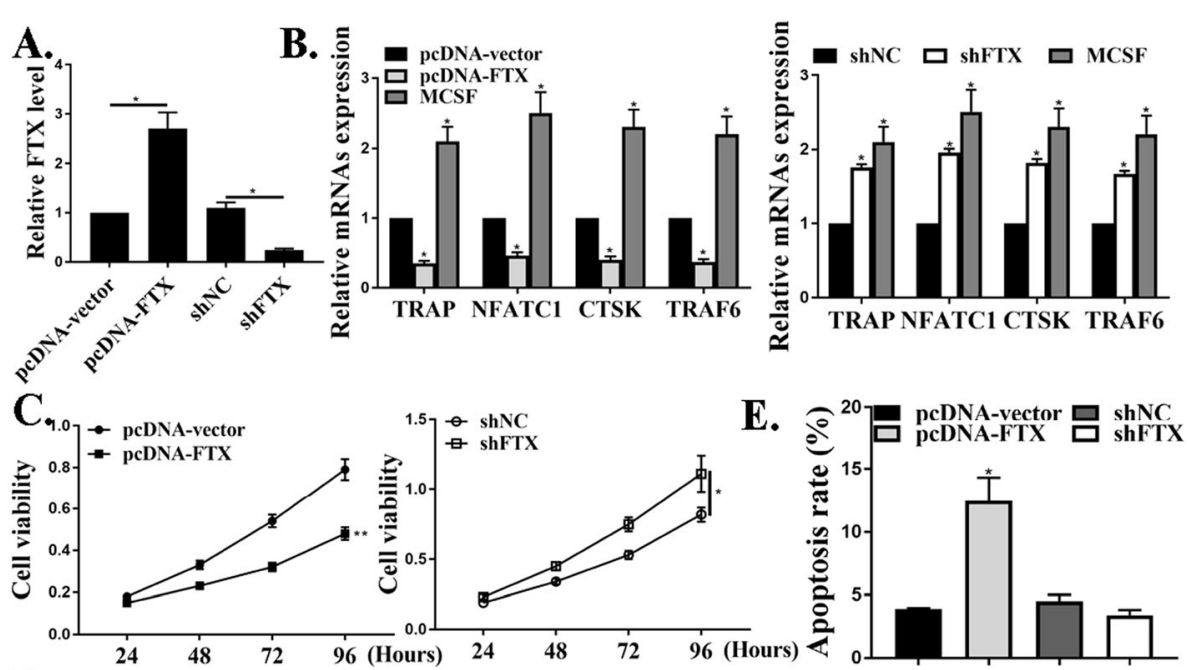

D.

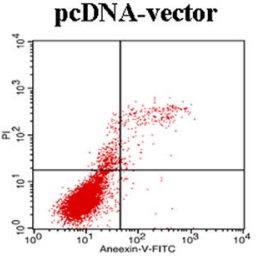

pcDNA-FTX

shNC
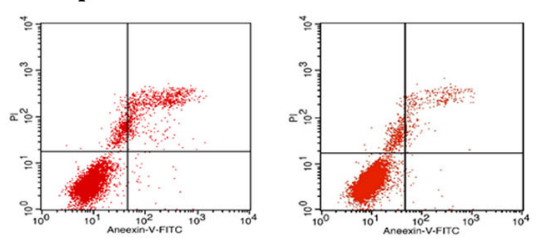

shFTX

F.
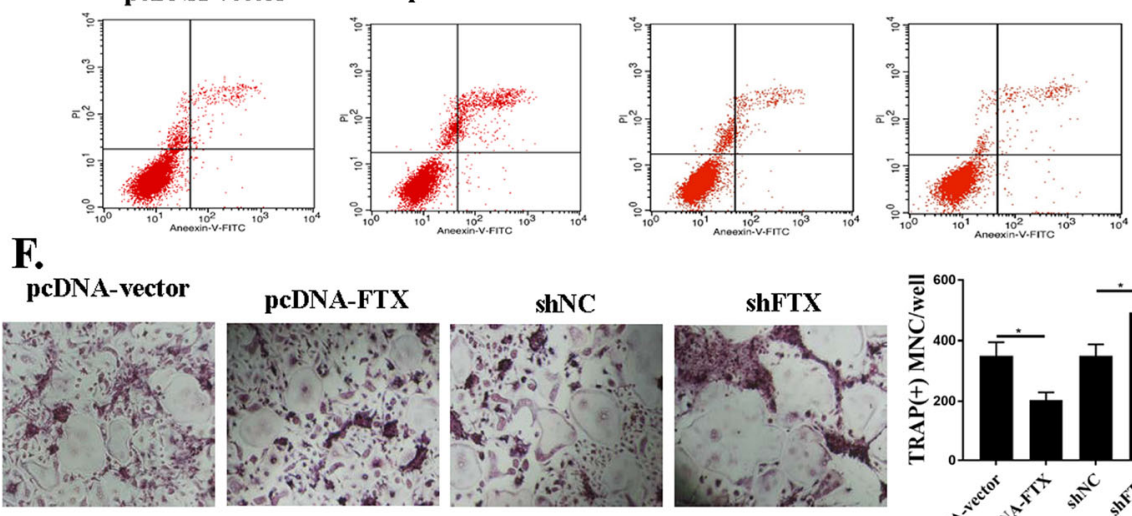

G.
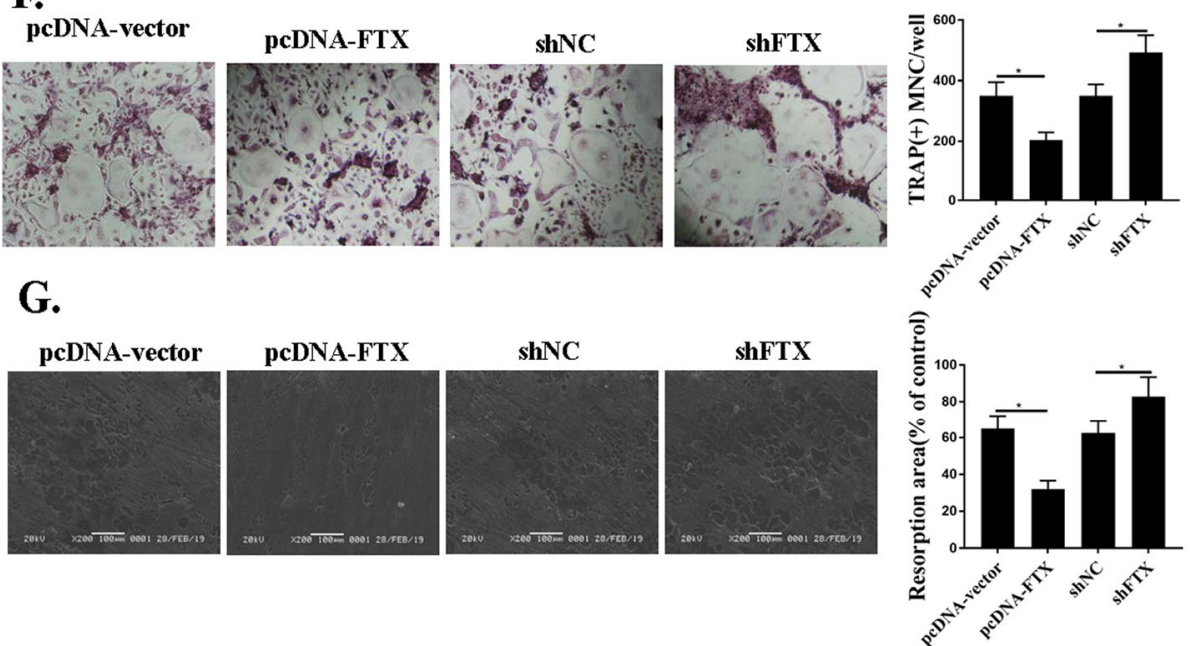

Fig. 2 Over-expressions of FTX inhibited osteoclast differentiation. a. FTX mRNA levels under pcDNA-vector or pcDNA-FTX. b. qRT-PCR results of the expressions of TRAP, CTSK, and NFATC1, TRAF6 under pCDNA-vector or pCDNA-FTX. $\mathbf{c}$, d and e. Cell viability and cell apoptosis of CD14 + PBMCs in transfection with pcDNAvector or pcDNA-FTX. f. TRAP-staining photos for osteoclast differentiation under transfection with pcDNA-vector or pcDNA-FTX. ${ }^{*} P<0.001 . n=3$

Aldrich, Tokyo, Japan). After removal of the TRAP fluid, the plate was washed three times with phosphatebuffered saline (PBS). TRAP-positive multinuclear cells were observed using an inverted microscope.

\section{Resorption pit assay}

Mature osteoclasts were generated by coculturing of mouse bone marrow cells and osteoblasts with VitD3 $(10 \mathrm{nM})$ on collagen gels for 6 days. The generated osteoclasts were replated on an Osteo Assay Surface plate
(Corning, NY, USA), allowed to settle for $2 \mathrm{~h}$, and then incubated with different concentrations of WESS for 24 $h$. After the incubation period, osteoclasts were visualized by TRAP staining. Resorption pits were photographed and analyzed by using Image J software, after removing cells by using sodium hypochlorite bleach.

\section{Western blotting}

Protein was separated by $10 \%$ SDS-PAGE (Thermo, USA) and sent to PVDF membrane (Millipore, USA). 


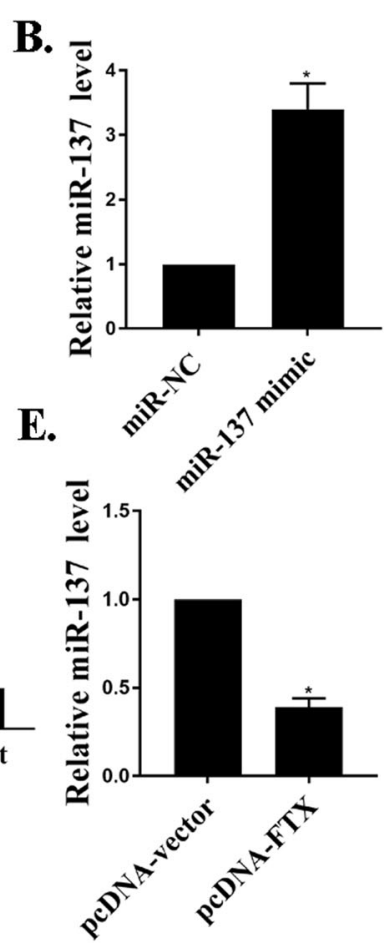

Fig. 3 miR-137 was one of the targets of FTX in CD14 + PBMCs. a. The binding sites between miR-137 and FTX by Starbase. b. The miR-137 mRNA. c-d. Luciferase reporter assays and RIP assays for the interactions between miR-137 and FTX. e. The miR-137 mRNA expressions under the transfection of FTX. ${ }^{*} p<0.001, n=3$

The membrane was blocked by $5 \%$ BSA. Next, we treated the membranes with anti-Notch1 (1:1000, Abcam, UK), anti-GAPDH (1:1000, beyotime, China) at $4{ }^{\circ} \mathrm{C}$ for a night and then HRP-anti-rabbit (1:1000, beyotime, China) for $2 \mathrm{~h}$ at $25 \mathrm{C}$. We detected the signals using the ECL (Bio-Rad Lab, USA).

\section{Flow cytometry}

After differentiation, cells were collected, washed and suspended. Then, it was incubated by Annexin $\mathrm{V}$ at $37^{\circ} \mathrm{C}$ for $10 \mathrm{~min}$, and stained by propidium iodide. Flow cytometer was employed to measure cell apoptosis.

\section{Statistical analysis}

All results were shown as mean \pm SD, analyzed by SPSS 21.0. Differences between groups were conducted by Student's t-test ( 2 groups) and one-way ANOVA ( 2 groups). $P$-value of $<0.05$ was considered to have significance.

\section{Results}

FTX was down-regulated and miR-137 was up-regulated in osteoporotic samples

The expressions of FTX in serum and bone tissues of OP patients were analyzed. The expressions of FTX in patients' serum $(n=30)$ were down-regulated compared with control $(n=21)$ (Fig. 1a). It is negatively related to the miR-137 expressions (Fig. 1b). In addition, the expressions of FTX in patients' bone tissues $(n=7)$ were down-regulated compared with control (non-OP) (Fig. 1c) $(n=7)$. miR-137 was up-regulated in OP patients' bone tissues (Fig. 1d).

\section{Over-expressions of FTX inhibited osteoclast differentiation}

pcDNA-FTX was efficiently over-expressed successfully in CD14+PBMCs, according to Fig. 2a. Figure 2b showed that osteoclast marker of TRAP, NFATC1, CTSK, and TRAF6 were hindered by FTX. Figures 2c-e demonstrated that the viabilities of CD14 + PBMCs were decreased by FTX, but cell apoptosis rate was promoted under FTX over-expressions. Figure $2 \mathrm{f}$ showed the experiments from TRAP-stained osteoclasts from each group, which revealed that pcDNA-FTX greatly inhibited osteoclast differentiation. It was obvious that FTX regulated osteoclast differentiation in CD14 + PBMCs.

miR-137 was one of the targets of FTX in CD14 + PBMCs

Figure 3a predicted the shared binding sites between FTX and miR-137 using bioinformatics. It also showed the relevant sequences of FTX-WT, miR-137, and FTXMUT. Figure $3 \mathrm{~b}$ investigated the successful transfection of miR-137 in cells. Next, we conducted the experiments to study the interactions between miR-137 and FTX in 


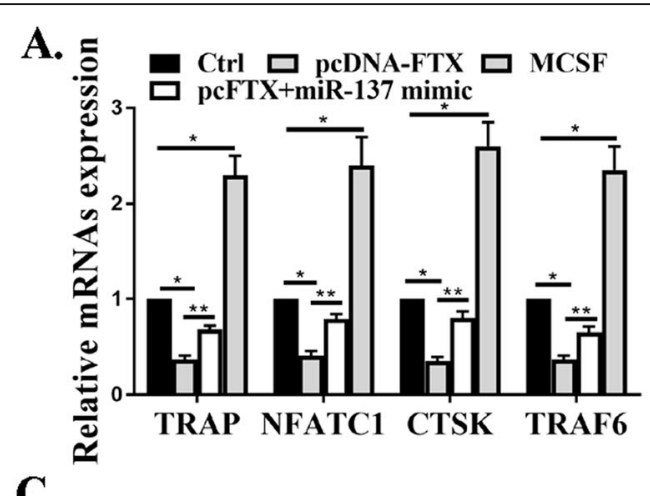

C.
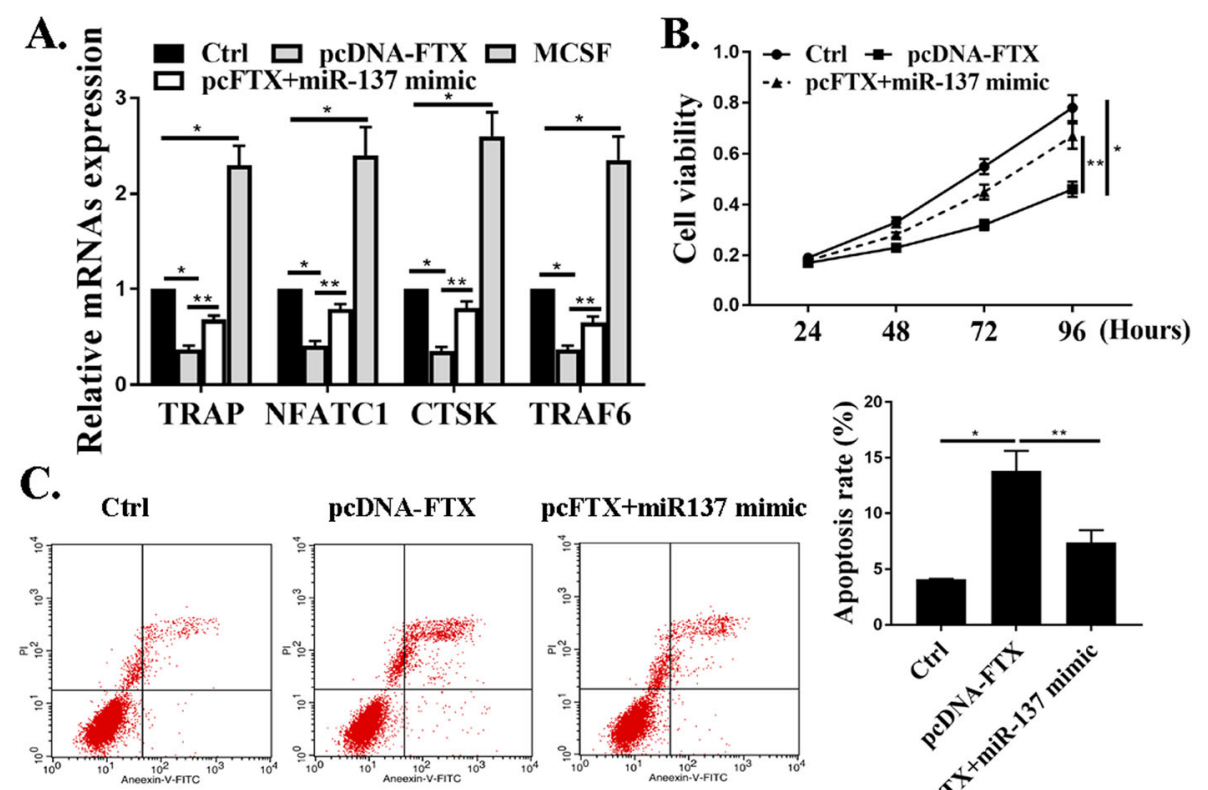

pcDNA-FTX

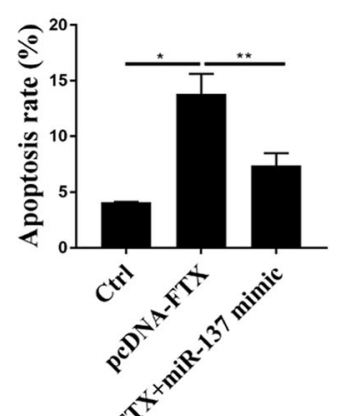

D. Ctrl

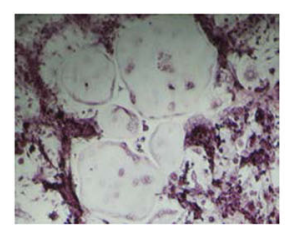

E.

Ctrl

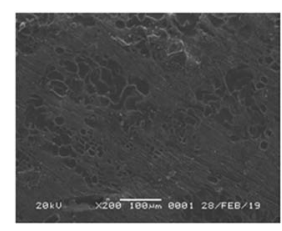

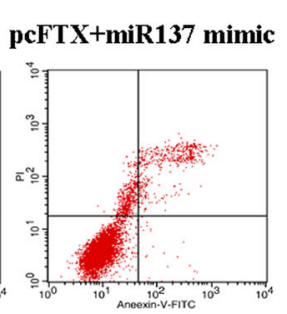

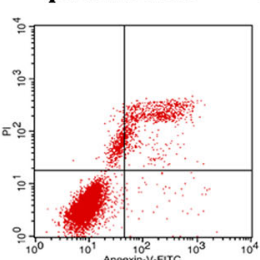

pcDNA-FTX
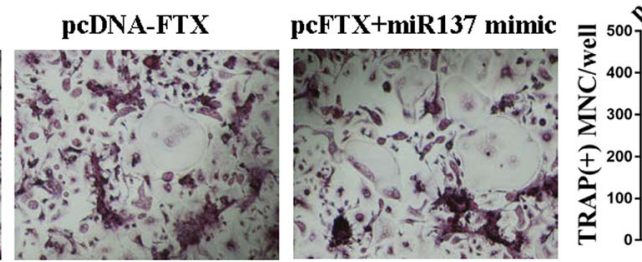
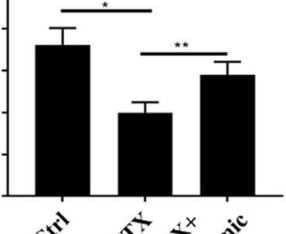

c

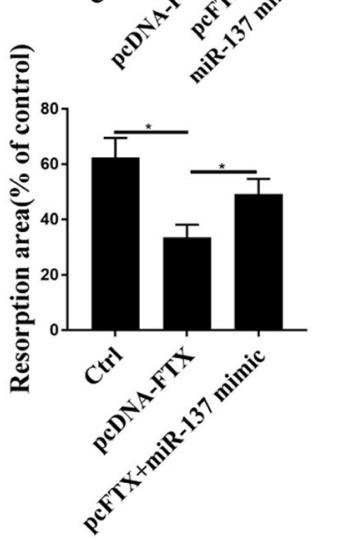

Fig. 4 miR-137 mediated the effects of FTX on osteoclast differentiation. a. qRT-PCR for expressions of TRAP, CTSK, and NFATC1, TRAF6 CD14 + PBMCs. $\mathbf{b}$ and $\mathbf{c}$. Cell viability and apoptosis by CCK-8 assays and flow cytometry. $\mathbf{d}$. TRAP-staining photos for osteoclast differentiation under transfection with pcDNA-FTX or pcFTX+miR-137 mimic. e. Representative microscopic pictures of resorption pit $P<0.001$. ${ }^{*} P<0.001, n=3$

OP by luciferase reporter assays and RIP assays in CD14 + PBMCs. RIP is a powerful method to investigate the physical associations between proteins and RNA molecules. Here, we utilized RIP experiment to verify the binding effect between FTX and miRNA. From Fig. 3c, miR-137 over-expressions resulted in a lower activity of FTX-WT. Figure 3d showed the RIP for binding effects between FTX and miR-137 with the AGO2 antibody. In contrast to the control IgG, higher FTX levels were found in the AGO2 antibody. Figure 3e revealed that over-expressions of FTX could down-regulate miR137 mRNA expressions in CD14 + PBMCs.

\section{miR-137 mediated the effects of FTX on osteoclast} differentiation

According to Fig. 4a, osteoclast-specific genes of TRAP, NFATC1, CTSK, and TRAF6 were all inhibited by FTX suppression, but this result was blunted by a miR-137 mimic. Figure $4 \mathrm{~b}$ and $\mathrm{c}$ revealed that the viabilities of CD14 + PBMCs were mitigated by FTX but the effects 


\section{A.}

Notch WT: 5' uuuuguuaacugauuugCAAUAa 3 '

| | | | |

miRNA : $3^{\prime}$ gaugcgcauaagaauucGUUAUu $5^{\prime}$

Notch MUT: 5' uuuguuaacugauuucCUUUUa 3 '

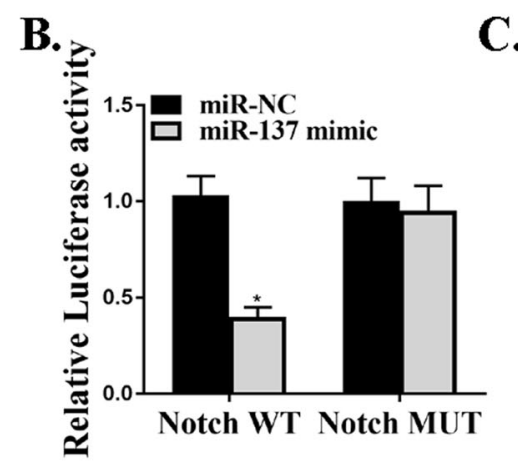

D.

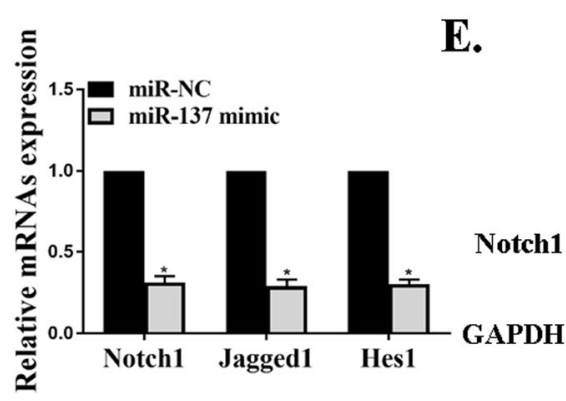

F.
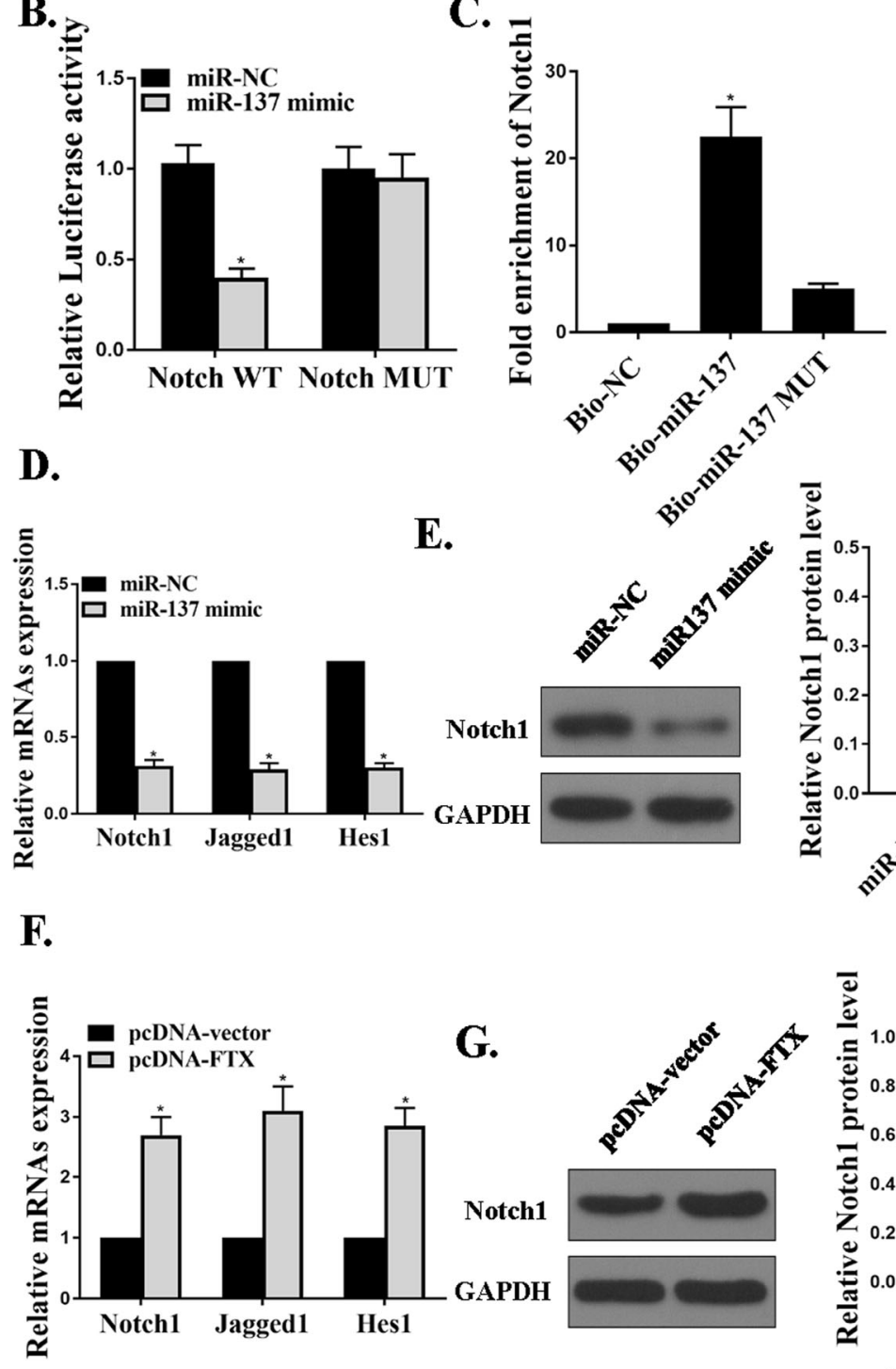

G.
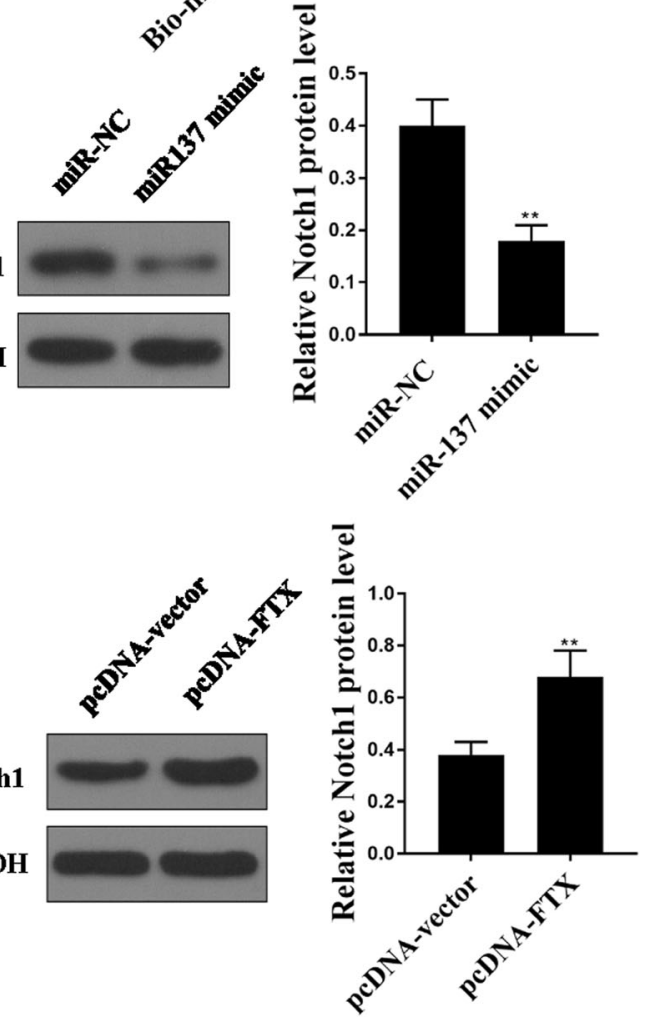

Fig. 5 MiR-137 regulated the Notch1 signaling pathways in CD14 + PBMCs. a. The binding sites in miR-137 and Noch1. b and c. Luciferase assays and RNA pull-down assays for interactions between miR-137 and Notch1. $\mathbf{d}$ and $\mathbf{e}$. The Notch1 mRNA and protein expressions under miR-137 mimic. $\mathbf{f}$ and $\mathbf{g}$. Notch 1 mRNA and protein expressions transfection of FTX. ${ }^{*} p<0.001,{ }^{* *} p<0.05, n=3$

were blunted by a miR-137 mimic. Cell apoptosis was promoted by FTX but the effects were blunted by a miR-137 mimic. Supplementary figure $1 \mathrm{~A}$ showed the apoptotic rate for early apoptotic cells (LR), and late apoptotic cells (UR). It was noticed that late apoptotic cells appeared under the transfection of pcDNA-FTX. However, this effect was also attenuated by miR-137 (supplementary figure 1B). Figure 4d showed the experiments from TRAPstained osteoclasts from each group, which revealed 

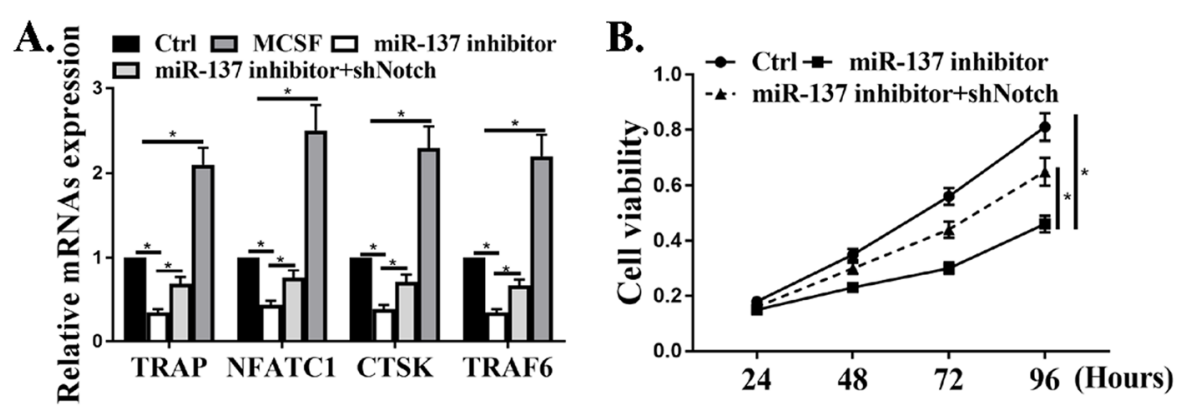

C. Ctrl
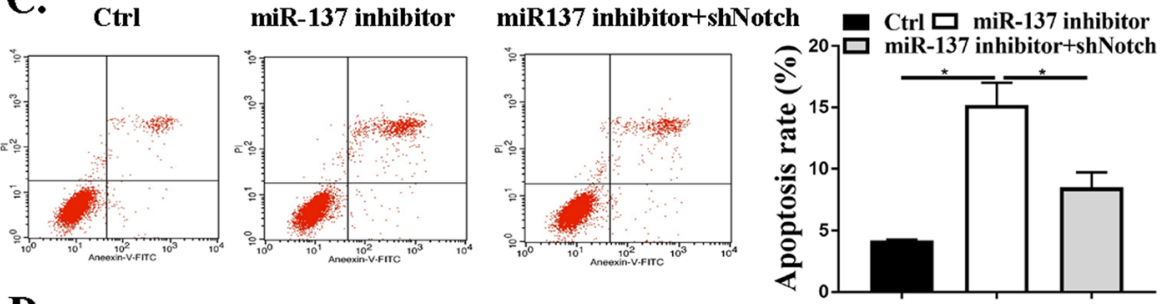

D.
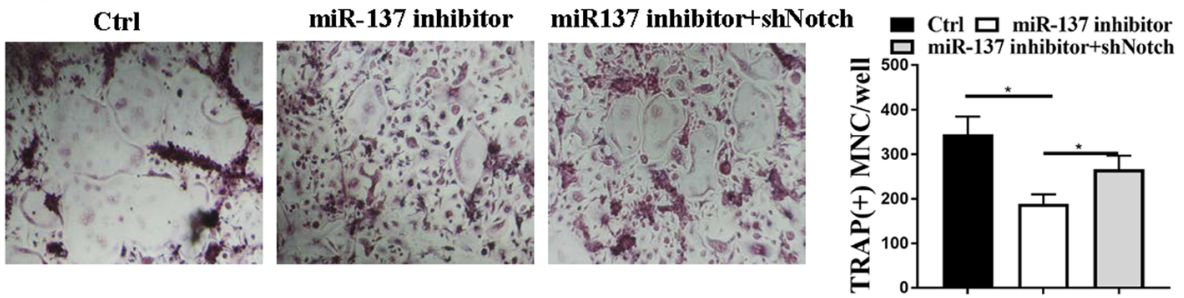

E.
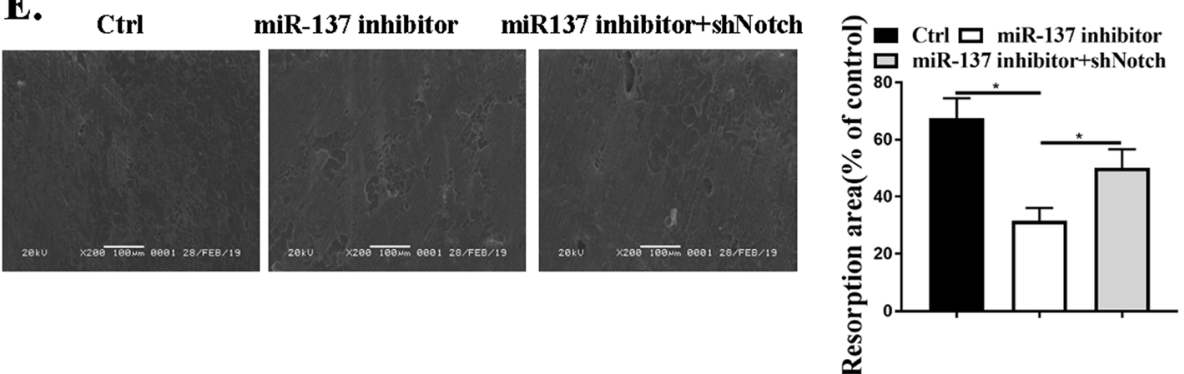

Fig. 6 The effects of miR-137 on osteoclast differentiation were mediated by Notch1 signaling pathways in CD14 + PBMCs. a. qRT-PCR for expressions of TRAP, CTSK, and NFATC1, TRAF6 CD14 + PBMCs. $\mathbf{b}$ and $\mathbf{c}$. Cell viability and apoptosis by CCK-8 assays and flow cytometry. $\mathbf{d}$. TRAPstaining photos for osteoclast differentiation under transfection with miR-137 inhibitor or miR-137 inhibitor+shNotch. e. Representative microscopic pictures of resorption pits. ${ }^{*} P<0.001$. ${ }^{*} P<0.001, n=3$

that pcDNA-FTX greatly inhibited osteoclast differentiation, while miR-137 mimic attenuated this effect. The data pointed out that FTX regulated osteoclast differentiation in CD14 + PBMCs.

\section{MiR-137 regulated the Notch1 signaling pathways in CD14 + PBMCs}

Figure 5a predicted the binding site in Notch1 and miR137 using bioinformatics. It showed the relevant binding sequences of Notch-WT, miR-137, and Notch-MUT. From Fig. 5b, miR-137 over-expressions resulted in the decreased luciferase activity of Notch1-WT. Figure $5 \mathrm{c}$ showed the pull-down assays. We found that Notch1 was pulled-down via bio-miR-137 but not bio-NC or bio-miR-137-Mut. Figure $5 \mathrm{~d}$ and e demonstrated that over-expressions of miR137 could down-regulate Notch1 mRNA and protein expressions in CD14 + PBMCs, but over-expressions of FTX could up-regulate Notch1 these expressions.

The effects of miR-137 on osteoclast differentiation were mediated by Notch1 signaling pathways in CD14 + PBMCs To further illustrate the mechanisms underlying miR137 and osteoclast differentiation, we evaluated the relative genes and cells in this process. The osteoclast-specific genes of TRAP, NFATC1, CTSK, and TRAF6 were all inhibited by miR137 suppression, but this result was 
blunted by shNotch (Fig. 6a). Furthermore, the viabilities of CD14 + PBMCs were mitigated by mirR-137 but the effects were blunted by a miR-137 mimic (Figs. 6b \& c). Cell apoptosis was promoted by FTX but the effects were blunted by shNotch. Figure $6 \mathrm{~d}$ showed the experiments from TRAP-stained osteoclasts from each group, which revealed that mir137 inhibitor greatly inhibited osteoclast differentiation, while shNotch attenuated this effect. Resorption pit assay also showed the same results (Fig. 6e.) The data pointed out that miR-137-notch regulated osteoclast differentiation in CD14 + PBMCs.

\section{Discussion}

Previous studies have established that OP probably leads to the dysregulation of some specific lncRNAs and miRNAs $[3,24,25]$. For example, Q. Wang et al. reported that the expressions of lncRNA MEG3 and miR-133a-3p were both up-regulated in OP samples [25]. Based on our qRT-PCR results, the expressions of FTX in patients' serum was suppressed compared with control. Besides, it is negatively related to the expressions of miR-137. In addition, the expressions of FTX in patients' bone tissues were suppressed compared with control (non-OP) and miR-137 was up-regulated in OP patients' bone tissues. For the first time, we propose that FTX was suppressed and miR-137 was upregulated in osteoporotic samples.

According to Q. Wang, the irregular expressions of related lncRNAs affect the osteogenic differentiation for OP patients [25]. Another study in 2018 also demonstrated that specific lncRNA could inhibit osteoclasts differentiation [23]. From our experiments, the osteoclast markers of TRAP, NFATC1, CTSK, and TRAF6 were all hindered by FTX. The viabilities of CD14 + PBMCs were decreased by FTX, but cell apoptosis rate was promoted under FTX over-expressions. It was obvious that FTX regulated osteoclast differentiation in CD14+ PBMCs. Similar to previous researches, we found that over-expressions of FTX inhibited osteoclast differentiation.

It was commonly known that lncRNAs and miRNAs could bind to each other and exert biological functions in multiple types of human cancers $[5,9]$. Firstly, our study from bioinformatics predicted the shared binding sites between FTX and miR-137. The luciferase reporter assays and RIP assays also confirmed the close interactions between miR-137 and FTX in CD14+PBMCs. Moreover, over-expressions of FTX could suppress miR137 mRNA expressions in CD14 + PBMCs. As far as we know, we are the first to report that miR-137 was directly targeted by FTX in CD14 + PBMCs.

The interactions between lncRNAs and miRNAs may reverse the biological effects resulted from lncRNAs [10, 27]. In consistence with previous studies, we noticed that osteoclast-specific genes of TRAP, NFATC1, CTSK, and TRAF6 were all inhibited by FTX suppression, but this result was blunted by a miR-137 mimic. Moreover, the viabilities of CD14+ PBMCs were mitigated by FTX but the effects were blunted by a miR-137 mimic. Cell apoptosis was promoted by FTX but the effects were blunted by a miR-137 mimic. Consistent with the well-established theories, our studies revealed that FTX regulated osteoclast differentiation in CD14+ PBMCs, while miR-137 mediated the effects of FTX on osteoclast differentiation.

A variety of researches have demonstrated the essential effects of a Notch signaling pathway in both bone remodeling $[1,19]$ and OP $[4,14]$. In our experiments, miR-137 over-expressions resulted in the decreased luciferase activity of Notch1-WT. Notch1 was pulled down by bio-miR-137 but not bio-NC or bio-miR-137-Mut. Over-expressions of miR-137 could suppress Notch1 mRNA and protein expressions in CD14+PBMCs, but over-expressions of FTX could up-regulate Notch1 these expressions. As a novel finding, miR-137 regulated the Notch1 signaling pathway in CD14 + PBMCs.

\section{Conclusion}

FTX was suppressed in OP samples and serums, however, miR-137 was greatly elevated. FTX reduced osteoclast-genesis and inhibited osteogenic differentiation by targeting miR-137, which inhibited the Notch1 signaling pathway. Our experiments and results pointed out that lncRNA FTX up-regulated miR-137 in OP by Notch1 signaling pathway.

\section{Supplementary information}

Supplementary information accompanies this paper at https://doi.org/10. 1186/s12891-020-03458-0.

Additional file 1: Figure S1. Apoptosis rate for UR and LR. A. The apoptotic rate for early apoptotic cells (LR), and late apoptotic cells (UR) under transfection of pcDNA-FTX and pcDNA-vector. B. The apoptotic rate for $L R$, and UR under transfection of pcDNA-FTX and pcFTX+miR-137 mimic.

Additional file 2.

Additional file 3 .

\section{Abbreviations}

OP: Osteoporosis; IncRNAs: Long non-coding RNAs; miRNAs: microRNAs

\section{Acknowledgments}

Not applicable.

Individual persons data

Not applicable.

Authors' contributions

YFY, PQY, ZKW, WWX contributed to data analysis, drafting or revising the article, gave final approval of the version to be published, and agree to be accountable for all aspects of the work". 


\section{Funding}

We thank the financial support from Dongguan social development project (2018507150241663)

\section{Availability of data and materials}

The analyzed data sets generated during the study are available from the corresponding author on reasonable request.

\section{Ethics approval and consent to participate}

The present study was approved by the Ethics Committee of the third People's Hospital of Dongguan City. The research has been carried out in accordance with the World Medical Association Declaration of Helsinki. All patients provided written informed consent prior to their inclusion within the study.

\section{Consent for publication}

Written informed consent was obtained from all individual participants included in the study.

\section{Competing interests}

The authors declare that they have no competing interests.

Received: 26 October 2019 Accepted: 24 June 2020

Published online: 13 July 2020

\section{References}

1. Bae Y, Yang T, Zeng H-C, Campeau PM, Chen Y, Bertin T, Dawson BC, Munivez E, Tao J, Lee BH. miRNA-34c regulates Notch signaling during bone development. Hum Mol Genet. 2012;21(13):2991-3000.

2. Chen HT, Lee MJ, Chen CH, Chuang SC, Chang LF, Ho ML, Hung SH, Fu YC, Wang YH, Wang HI. Proliferation and differentiation potential of human adipose-derived mesenchymal stem cells isolated from elderly patients with osteoporotic fractures. J Cell Mol Med. 2012;16(3):582-92.

3. Dou C, Cao Z, Yang B, Ding N, Hou T, Luo F, Kang F, Li J, Yang X, Jiang H. Changing expression profiles of IncRNAs, mRNAs, circRNAs and miRNAs during osteoclastogenesis. Sci Rep. 2016;6:21499.

4. Fan J-Z, Yang L, Meng G-L, Lin Y-s, Wei B-Y, Fan J, Hu H-M, Liu Y-W, Chen S, Zhang J-K. Estrogen improves the proliferation and differentiation of hBMSCs derived from postmenopausal osteoporosis through notch signaling pathway. Mol Cell Biochem. 2014;392(1-2):85-93.

5. Fei $Q$, Bai $X$, Lin J, Meng H, Yang $Y$, Guo A. Identification of aberrantly expressed long non-coding RNAs in postmenopausal osteoporosis. Int J Mol Med. 2018;41(6):3537-50.

6. Gao Y, Xiao F, Wang C, Wang C, Cui P, Zhang X, Chen X. Long noncoding RNA MALAT1 promotes osterix expression to regulate osteogenic differentiation by targeting miRNA-143 in human bone marrow-derived mesenchymal stem cells. J Cell Biochem. 2018;1 19(8):6986-96.

7. Guo X-B, Hua Z, Li C, Peng L-P, Wang J-S, Wang B, Zhi Q-M. Biological significance of long non-coding RNA FTX expression in human colorectal cancer. Int J Clin Exp Med. 2015:8(9):15591.

8. Hao L, Fu J, Tian Y, Wu J. Systematic analysis of IncRNAs, miRNAs and mRNAs for the identification of biomarkers for osteoporosis in the mandible of ovariectomized mice. Int J Mol Med. 2017;40(3):689-702.

9. Huang $Y$, Zheng $Y$, Jin C, Li X, Jia L, Li W. Long non-coding RNA H19 inhibits adipocyte differentiation of bone marrow mesenchymal stem cells through epigenetic modulation of histone deacetylases. Sci Rep. 2016;6: 28897.

10. Jalali S, Bhartiya D, Lalwani MK, Sivasubbu S, Scaria V. Systematic transcriptome wide analysis of IncRNA-miRNA interactions. PLoS One. 2013; 8(2):e53823.

11. Jin D, Wu X, Yu H, Jiang L, Zhou P, Yao X, Meng J, Wang L, Zhang M, Zhang Y. Systematic analysis of IncRNAs, mRNAs, circRNAs and miRNAs in patients with postmenopausal osteoporosis. Am J Transl Res. 2018;10(5):1498.

12. Kanis JA, Melton LJ III, Christiansen C, Johnston CC, Khaltaev N. The diagnosis of osteoporosis. J Bone Miner Res. 1994;9(8):1137-41.

13. Klibanski A, Adams-Campbell L, Bassford TL, Blair SN, Boden SD, Dickersin K, Gifford DR, Glasse L, Goldring SR, Hruska K. Osteoporosis prevention, diagnosis, and therapy. J Am Med Assoc. 2001;285(6):785-95.

14. Li Y, Li S, Gao Y, Li J, Zhang B. Crucial role of notch signaling in osteogenic differentiation of periodontal ligament stem cells in osteoporotic rats. Cell Biol Int. 2014;38(6):729-36.
15. Lin GL, Hankenson KD. Integration of BMP, Wnt, and notch signaling pathways in osteoblast differentiation. J Cell Biochem. 2011;112(12):3491501.

16. Liu M, Lang N, Qiu M, Xu F, Li Q, Tang Q, Chen J, Chen X, Zhang S, Liu Z. miR-137 targets $\mathrm{Cdc} 42$ expression, induces cell cycle $\mathrm{G} 1$ arrest and inhibits invasion in colorectal cancer cells. Int J Cancer. 2011;128(6):1269-79.

17. Long B, Li N, Xu X-X, Li X-X, Xu X-J, Guo D, Zhang D, Wu Z-H, Zhang S-Y. Long noncoding RNA FTX regulates cardiomyocyte apoptosis by targeting miR-29b-1-5p and Bcl212. Biochem Biophys Res Commun. 2018;495(1):312-8.

18. Rachner TD, Khosla S, Hofbauer LC. Osteoporosis: now and the future. Lancet. 2011;377(9773):1276-87.

19. Regan J, Long F. Notch signaling and bone remodeling. Current Osteoporosis Rep. 2013;11(2):126-9.

20. Silber J, Lim DA, Petritsch C, Persson Al, Maunakea AK, Yu M, Vandenberg SR, Ginzinger DG, James CD, Costello JF. miR-124 and miR-137 inhibit proliferation of glioblastoma multiforme cells and induce differentiation of brain tumor stem cells. BMC Med. 2008;6(1):14.

21. Sørensen MG, Henriksen K, Schaller S, Henriksen DB, Nielsen FC, Dziegiel $\mathrm{MH}$, Karsdal MA. Characterization of osteoclasts derived from CD14+ monocytes isolated from peripheral blood. J Bone Miner Metab. 2007;25(1): 36-45.

22. Sun G, Ye P, Murai K, Lang M-F, Li S, Zhang H, Li W, Fu C, Yin J, Wang A. miR-137 forms a regulatory loop with nuclear receptor TLX and LSD1 in neural stem cells. Nature Commun. 2011;2:529.

23. Tang Z, Gong Z, Sun X. LncRNA DANCR involved osteolysis after total hip arthroplasty by regulating FOXO1 expression to inhibit osteoblast differentiation. J Biomed Sci. 2018;25(1):4.

24. Tong X, P-c G, Xu S-z, Lin X-j. Long non-coding RNA-DANCR in human circulating monocytes: a potential biomarker associated with postmenopausal osteoporosis. Biosci Biotechnol Biochem. 2015;79(5):732-7.

25. Wang Q, Li Y, Zhang Y, Ma L, Lin L, Meng J, Jiang L, Wang L, Zhou P, Zhang $Y$. LncRNA MEG3 inhibited osteogenic differentiation of bone marrow mesenchymal stem cells from postmenopausal osteoporosis by targeting miR-133a-3p. Biomed Pharmacother. 2017;89:1178-86.

26. Wu X-B, Li Y, Schneider A, Yu W, Rajendren G, labal J, Yamamoto M, Alam $M$, Brunet $L$, Blair HC. Impaired osteoblastic differentiation, reduced bone formation, and severe osteoporosis in noggin-overexpressing mice. J Clin Invest. 2003;112(6):924-34.

27. Yoon J-H, Abdelmohsen $\mathrm{K}$, Gorospe M. Functional interactions among microRNAs and long noncoding RNAs. Elsevier; 2014. p. 9-14.

28. Zhao Q, Li T, Qi J, Liu J, Qin C. The miR-545/374a cluster encoded in the Ftx IncRNA is overexpressed in HBV-related hepatocellular carcinoma and promotes tumorigenesis and tumor progression. PLoS One. 2014;9(10): e109782.

29. Zhu X, Li Y, Shen H, Li H, Long L, Hui L, Xu W. miR-137 inhibits the proliferation of lung cancer cells by targeting Cdc42 and Cdk6. FEBS Lett. 2013;587(1):73-81.

\section{Publisher's Note}

Springer Nature remains neutral with regard to jurisdictional claims in published maps and institutional affiliations.

Ready to submit your research? Choose BMC and benefit from:

- fast, convenient online submission

- thorough peer review by experienced researchers in your field

- rapid publication on acceptance

- support for research data, including large and complex data types

- gold Open Access which fosters wider collaboration and increased citations

- maximum visibility for your research: over $100 \mathrm{M}$ website views per year

At $\mathrm{BMC}$, research is always in progress.

Learn more biomedcentral.com/submissions 